\title{
Marges Linguistiques 5, M.L.M.S. éditeur
}

\section{Sara Vecchiato}

\section{(2) OpenEdition}

\section{Journals}

\section{Édition électronique}

URL : https://journals.openedition.org/studifrancesi/41425

DOI : 10.4000/studifrancesi.41425

ISSN : 2427-5856

\section{Éditeur}

Rosenberg \& Sellier

\section{Édition imprimée}

Date de publication : 1 juillet 2004

Pagination : 239

ISSN : 0039-2944

\section{Référence électronique}

Sara Vecchiato, « Marges Linguistiques 5, M.L.M.S. éditeur », Studi Francesi [En ligne], 142 (XLVIII | I) | 2004, mis en ligne le 30 novembre 2015, consulté le 09 septembre 2021. URL : http://

journals.openedition.org/studifrancesi/41425; DOI : https://doi.org/10.4000/studifrancesi.41425

Ce document a été généré automatiquement le 9 septembre 2021.

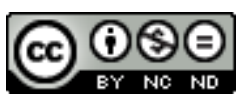

Studi Francesi è distribuita con Licenza Creative Commons Attribuzione - Non commerciale - Non opere derivate 4.0 Internazionale. 


\title{
Marges Linguistiques 5, M.L.M.S. éditeur
}

\author{
Sara Vecchiato
}

\section{RÉFÉRENCE}

MARGES LINGUISTIQUES 5 (2003) M.L.M.S. éditeur, Saint-Chamas

1 C'est Daniel véRoniQue qui a dirigé le cinquième numéro de cette revue électronique, consacré au thème: «Enjeux des acquisitions grammaticales et discursives en langue étrangère». Nonobstant le titre générique, toutes les contributions sont axées sur le français, sauf celle de Patrizia GIULIANO, Antonella D'AMBRosio et Paolo GRECo, qui traite de l'italien (L'expression des relations spatiales en Italien langue première et langue seconde), et celle de S.E. CARROLL, qui analyse le rôle de la correction dans l'acquisition des langues à travers l'étude d'un cas d'acquisition de la négation en anglais (Comment est-ce qu'on interprète: «Ne dites pas... mais dites plutôt ...»?).

Florence MYLES analyse le développement narratif en français langue étrangère. Elle compare la performance d'apprenants débutants effectuant la même activité à un an d'intervalle. Il est suggéré que les étapes développementales identifiées sont dues à la difficulté d'établir des liens au moyen de processus morphosyntactiques ou discursifs entre différents éléments dans la phrase ou le récit, liens qui seront d'abord réalisés dans des domaines locaux. L'analyse discursive de leurs récits montre que les apprenants débutants ont recours à des schémas narratifs typiques d'enfants préscolaires (Les premières étapes du développement narratif en français langue étrangère: une étude longitudinale).

3 Mariane STARREN procède à une analyse de l'acquisition rudimentaire de la temporalité par des Marocains apprenant le français. L'auteur démontre qu'il y a des raisons internes au niveau de l'organisation du discours qui conduisent l'apprenant à la grammaticalisation d'outils linguistiques dans les références temporelles. Le nonrespect de trois principes pragmatiques fondamentaux bien définis du discours, qui 
assurent la cohérence temporelle dans les contextes diagnostiques, semble conduire les apprenants à mettre en place un marquage grammatical de l'aspect. Les conclusions de cette étude indiquent que la dynamique de l'acquisition de la langue (seconde) se situe à l'interface de l'organisation du discours et des outils linguistiques à la disposition de l'apprenant (La cohérence temporelle comme facteur de développement de l'aspect grammatical en français L2).

Cyrille GRANGET étudie l'appropriation des expressions lexicales de la temporalité chez des apprenants germanophones du français L2. L'auteur nous montre quelles catégories conceptuelles sont prioritairement exprimées au moyen des expressions lexicales de la temporalité, et selon quelle échelle implicationnelle les différentes composantes fonctionnelles de ces expressions formelles sont acquises. Il apparaît ainsi que l'expression lexicale de la succession précède celle de l'itérativité et qu'il existe un stade initial durant lequel les adverbes «de succession» jouent un rôle de structuration temporelle du récit tout en étant dépourvus de fonction sémantique de postériorité (Appropriation des fonctions discursive et sémantiques de quelques expressions lexicales de la temporalité en français L2).

5 Sun JILI s'occupe de l'acquisition de la morphologie verbale du français L2 par les apprenants sinophones. Premièrement, chez les débutants, on constate l'apparition précoce et l'emploi massif de la forme du présent de l'indicatif. Deuxièmement, on observe le processus du développement de la morphologie. On dégage un ordre d'émergence de différentes formes morphologiques: présent - passé composé - imparfait plus-que-parfait; on constate aussi un décalage entre l'acquisition de la forme et sa fonction, illustré par l'enrichissement des valeurs aspecto-temporelles des mêmes formes. Troisièmement, JiLI étudie l'influence de la L1 par le biais de l'analyse de l'usage des formes morphologiques propres aux sinophones (Comment se développe la morphologie verbale en français $L 2$ chez les sinophones?).

Monique LAMBERT compare des organisations de textes narratifs chez des enfants francophones âgés de 4, 7 et 10 ans et chez des apprenants polonais de niveau débutant et avancé. Les textes sont comparés aux récits de natifs francophones et polonophones adultes dans la même tâche. Les variables retenues relatives à la cohésion sont les modes de (ré)introduction des référents et leur maintien d'un énoncé à l'autre. Pour la connexité, on examine les liaisons interénoncés, et notamment le degré d'explicitation des relations causales et temporelles (Cohésion et connexité dans des récits d'enfants francophones et d'apprenants polonophones du français).

7 Maarit MUTTA examine ce que recouvre la réflexion dans le domaine de l'apprentissage de langues secondes chez des étudiants finlandais, par l'intermédiaire d'un test à trous. Ce test mesure la maîtrise du vocabulaire et des structures grammaticales, ainsi que la saisie du sens de l'ensemble du texte. Il ressort que l'amélioration des connaissances lexicales favorisent l'augmentation de la compréhension. Les structures familières, apprises très tôt à l'école, semblent être automatisées, et cette automatisation semble diminuer l'incertitude linguistique dans les réponses. En outre, les étudiants profitent de l'input multiple et varié, même dans le milieu non naturel qu'est la classe (Activité métalinguistique chez des locuteurs non natifs).

8 Miriam BEN-RAFAeL considère le jeu discours /grammaire, dans deux situations de contact. Il examine (i) l'interlangue de lycéens hébréophones acquérant le français L2 en milieu guidé; (ii) le discours de locuteurs adultes francophones $\mathrm{Fr} / \mathrm{L} 1$ qui ont acquis l'hébreu/L2 lors de leur immigration en Israël. Des corpus oraux et écrits permettent 
d'observer, d'une part la mise en place d'une grammaire à travers des pratiques discursives dans les contextes considérés, et d'autre part le rôle décisif de la grammaire L1 dans la formation d'un nouveau vernaculaire franbréophone. Ces deux cas confirment que la grammaire constitue effectivement l'élément fort de la langue (Pratique discursive et mise en place grammaticale).

Martine FARACO cherche à observer quelle(s) implication(s) peut avoir la prosodie dans l'apprentissage/acquisition grammatical et discursif en langue seconde. Si la prosodie peut se manifester par différents moyens phonétiques, il faut essayer d'en dégager l'interface phonologique. L'objectif de l'auteur est de chercher des moyens de transcrire localement la prosodie dans les corpus oraux issus de la classe de langue et donc, globalement, d'impliquer davantage phonétique et phonologie à la réflexion sur l'apprentissage/acquisition d'une langue seconde (Intonation et apprentissages grammatical et discursif en classe de langue: observations empiriques).

10 Stephan PIRSCH analyse la syntaxe de la négation comme phénomène d'acquisition. Il observe le processus d'acquisition du système négatif français par Berta, une immigrante du Chili qui commence l'acquisition à l'âge de trente ans. PIRSCH propose que Berta, au cours du processus d'acquisition, recourt en même temps à deux structures différentes. L'une est plutôt rudimentaire, comparable aux premières étapes enfantines, tandis que l'autre correspond au système de la langue cible. Ce phénomène s'explique par la structure phonétique de la langue maternelle de Berta (L'acquisition de la syntaxe de la négation: quelques indices de l'utilisation de deux grammaires simultanées par une apprenante d'une seconde langue).

Daniel VÉRONIQUE et Henriette STOFFEL fournissent une description des itinéraires d'appropriation de la négation en français L2 par 6 adultes marocains. Deux marques de la négation sont privilégiés: non et pas. L'emploi de la première marque semble être déterminé par des facteurs pragmatiques, alors que l'usage du deuxième négateur est davantage déterminé par des considérations grammaticales. Les informateurs présentent une relative diversité de parcours dans l'appropriation de la négation en L2. L'étude n'est pas en mesure de conclure à une influence décisive de la L1 dans l'emploi de la négation en L2 (L'acquisition de la négation en français par des adultes arabophones).

Tsuyoshi KIDA aborde la question de compréhension du discours par les locuteurs non natifs et d'exploiter l'implication de l'information visuelle au sein de l'interaction. La méthode adoptée est une comparaison des interactions entre la condition en face à face et la condition de non-visibilité mutuelle. Les informateurs principaux sont 7 Japonais ayant le français comme langue seconde. Les points traités sont la focalisation, la projection, l'ellipse, la métaphore, l'anaphore, la syntaxe, les relations discursives et le domaine extra discursif en rapport avec les données visuelles. L'analyse suggère que l'information visuelle est constitutive du discours (Le rôle de l'information visuelle dans la compréhension discursive en $\mathrm{L}$ 2). 